\title{
Drogas procinéticas não devem ser prescritas rotineiramente para o tratamento de doença do refluxo gastroesofágico em Pediatria
}

\author{
Prokinetics should not be routinely prescribed in the treatment of gastroesophageal reflux disease \\ in pediatric patients
}

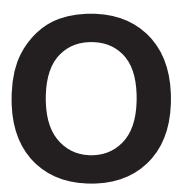

artigo de Magalhães et al ${ }^{(1)}$ intitulado Revisão sistemática e metanálise do uso de procinéticos no refluxo gastroesofágico e na doença do refluxo gastroesofágico em Pediatria é de extrema importância para o pediatra, pois essas entidades são de elevada prevalência nas distintas faixas etárias, desde o período neonatal até a adolescência. Devido às múltiplas facetas que envolvem o diagnóstico diferencial e a abordagem terapêutica da doença do refluxo gastroesofágico, inúmeras revisões de literatura e constantes atualizações são publicadas pelas diferentes sociedades de pediatria, gastroenterologia, pneumologia e otorrinolaringologia ${ }^{(2,3)}$.

Os sintomas de Doença do Refluxo Gastroesofágico (DRGE) são menos frequentes do que os sintomas de refluxo gastroesofágico (RGE), mas, ainda assim, são muito prevalentes. Estima-se que aproximadamente 2\% das crianças com idade entre três e nove anos e 5 a $8 \%$ entre dez e 17 anos apresentem sensação de queimação retroesternal e regurgitação ácida de forma intermitente ${ }^{(4)}$. A queimação retroesternal isolada pode ser detectada em $17,8 \%$ dos adolescentes. No mundo ocidental, a prevalência de DRGE é elevada na idade adulta e estimada em frequências acima de 10\% em inúmeras publicações ${ }^{(4,5)}$.

As crianças portadoras de DRGE necessitam de terapêutica medicamentosa específica, utilizada muitas vezes durante anos, com o objetivo básico de reduzir a morbidade e as eventuais complicações. A condução terapêutica é de difícil execução na prática diária e pode, em muitos casos, suscitar dúvidas quanto ao manuseio tanto de lactentes quanto de crianças maiores, como enfatizado por Deal et $a^{(/ 6)}$.

O subdiagnóstico da doença põe a criança em risco para a ocorrência de complicações graves, como broncoespasmo, esofagite, estenose esofagiana e esôfago de Barrett. Todavia, a valorização demasiada da sintomatologia, principalmente quanto à presença somente de regurgitação, pode levar o médico a excessos de diagnóstico e tratamento ${ }^{(7)}$.

A Sociedade Norteamericana de Gastroenterologia, Hepatologia e Nutrição (NASPGHAN) publicou, em $2001^{(8)}$, um guia de avaliação diagnóstica e manuseio terapêutico do refluxo gastresofágico na infância, no qual os autores revisaram as medidas posturais, dietéticas e medi- camentosas a serem utilizadas. Nessa ocasião, a medida terapêutica inicial consistia no emprego de droga procinética, a cisaprida, para todas as faixas etárias. Não há, nesse artigo, nenhuma referência quanto ao uso dos demais procinéticos, tais como a metoclopramida, a domperidona ou o betanecol.

A cisaprida é um agente serotoninérgico que libera grande quantidade de acetilcolina nas sinapses dos plexos mioentéricos da parede intestinal. A cisaprida apresenta efeitos procinéticos no esfíncter esofágico inferior e no estômago, diminuindo o tempo de esvaziamento gástrico, o que concorre para a diminuição dos episódios de refluxo gastroesofágico. Dentre as drogas usadas no tratamento da DRGE na criança, certamente a cisaprida é a mais estudada em ensaios clínicos controlados e randomizados ${ }^{(9)}$.

A domperidona é um antagonista periférico do receptor D2 da dopamina. A propriedade básica da mesma consiste em diminuir o tempo de refluxo pós-prandial e é fundamentalmente usada para o controle das regurgitações e vômitos. Desde a suspensão da comercialização da cisaprida, a domperidona passou a ser muito usada, especialmente em nosso meio. Há poucos ensaios clínicos que utilizam domperidona para o tratamento do RGE em crianças $^{(10)}$.

A revisão publicada nesse fascículo reúne apenas quatro estudos que empregam a domperidona no tratamento de RGE em crianças. Esses estudos envolveram um total de 126 pacientes e foram publicados entre os anos de 1979 e 1994. A metanálise aponta vantagem para a domperidona em relação ao placebo no final do estudo $(n=126 ; R R=0,27 ; \mathrm{IC} 95 \%=0,14-0,52 ; p<0,001)^{(1)}$. Analisando as publicações isoladamente, observam-se dados que suscitam dúvidas e merecem reflexão.

Na publicação de Clara, em 1979(11), acompanharam-se 32 crianças com idades entre 2,5 meses e dez anos, com história de regurgitações e vômitos e nenhuma descrição efetiva de doença do refluxo. A intervenção nas primeiras duas semanas utilizou dose de $0.3 \mathrm{mg} / \mathrm{kg}$ em três tomadas ao dia, porém metade dos 14 pacientes que receberam domperidona não apresentou melhora dos sintomas, passando a receber, nas duas semanas subsequentes, a dose de $0,6 \mathrm{mg} / \mathrm{kg}$ em três tomadas ao dia. Apenas com a dose duplicada a domperidona demonstrou eficácia 
na redução dos sintomas: 93\% para o Grupo Medicação contra $33 \%$ no Grupo Placebo. Este é exatamente o estudo que apresenta o melhor resultado na metanálise publicada por Magalhães et $a^{\left({ }^{(1)}\right.}$. O segundo estudo em que a domperidona demonstrou eficácia terapêutica foi publicado por de Loore, em 1979(12), abrangendo 47 crianças entre três semanas de vida e sete anos, com história de regurgitações e vômitos e diagnóstico apenas clínico de RGE. Comparouse a ação da domperidona $0,3 \mathrm{mg} / \mathrm{kg}$ e metoclopramida $0,3 \mathrm{mg} / \mathrm{kg}$ frente ao Grupo Placebo por um período de apenas duas semanas. Os resultados demonstraram $73 \%$ de ausência de vômitos para o grupo domperidona, 43\% para metoclopramida e $7 \%$ para o Grupo Placebo. A mediana de idade para o grupo domperidona foi de nove meses e para o Grupo Metoclopramida, seis meses. A amplitude e heterogeneidade quanto à faixa etária das amostras populacionais avaliadas podem ter influenciado o resultado final, uma vez que há uma tendência à resolução espontânea dos casos de regurgitações e vômitos no segundo semestre de vida.

Nelson et al, em 1997(5), acompanharam aproximadamente 1.000 lactentes saudáveis no primeiro ano de vida, constatando que mais de $70 \%$ deles regurgitam frequentemente $\mathrm{e}$, às vezes, vomitam nos primeiros meses de vida. A partir do segundo semestre, aproximando-se do final do primeiro ano, apenas $10 \%$ dos lactentes normais persistem com regurgitações.

O trabalho randomizado duplo-cego placebo controlado de Bines et al, em 1992 $2^{(13)}$, incluiu 17 pacientes com idades variando entre cinco meses e 11,3 anos. O diagnóstico em questão foi confirmado por dados clínicos, radiológicos e prova de pHmetria esofágica. Utilizou-se a dose de domperidona de $0,6 \mathrm{mg} / \mathrm{kg} /$ dia fracionada em três tomadas durante quatro semanas, não se detectando melhora do quadro sintomatológico nos grupos estudados. Houve uma redução do número de episódios de refluxos ácidos pósprandiais na ordem de $25 \%$ no Grupo Domperidona, porém não foram descritos os dados de pHmetria no período além das duas horas pós-refeição. O número total de refluxos foi 69 no Grupo Domperidona e 16 no Grupo Placebo. Alguns dados metodológicos devem ser considerados: a idade média dos pacientes do Grupo Domperidona foi de 3,6 anos e a do grupo placebo de 2,4 anos, sendo este um fator que pode interferir no número total de refluxos registrados. A amostragem total incluiu apenas 17 participantes, número muito reduzido, o que não confere poder de amostragem suficiente para análise satisfatória.

Carrocio et al, em 1993 ${ }^{(14)}$, publicaram um estudo envolvendo 80 lactentes com idade entre um e 18 meses, com RGE confirmado por prova radiológica e pHmetria esofágica de 24 horas. Os pacientes foram seguidos por oito semanas e distribuídos em quatro grupos; domperidona $0,3 \mathrm{mg} / \mathrm{kg}$; domperidona $0,3 \mathrm{mg} / \mathrm{kg}$ mais hidróxido de alumínio e magnésio; domperidona mais alginato e um Grupo Placebo. Os pacientes apresentavam sintomatologia expressiva, porém não descrita com maiores detalhes. O Grupo Domperidona mais Alginato apresentou redução do número de refluxos ácidos de 59 para 48 no final do estudo ( $p<0,009$ ), enquanto no Grupo Placebo constatou-se médias de 65 e 68 refluxos, respectivamente. Ao comparar a porcentagem do tempo de refluxo (índice total de refluxo durante a prova) dos Grupos Domperidona à do Grupo Placebo, não houve melhora significativa dos pacientes que receberam a medicação. Os autores igualmente não detectaram alterações quanto ao cortejo sintomatológico.

Pritchard et al, em 2005 ${ }^{(10)}$, revisaram estes dados e constataram apenas evidências menores da eficácia da domperidona para reduzir os sintomas de refluxo em crianças. Os autores apontam, igualmente, que não há dados que demonstrem uma clara ação da droga em casos de doença do refluxo decorrente de esofagite confirmada por exame endoscópico.

A domperidona tem pouca ação sobre o sistema nervoso central, porém são descritos raros casos em lactentes de alterações neurológicas, com sintomas extrapiramidais e episódios de movimentos oculógiros. Tal como a cisaprida, a domperidona também é metabolizada no sistema enzimático hepático citocromo P450. Assim, o nível sérico da droga pode aumentar se houver uso concomitante de derivados imidazólicos e antibióticos macrolídeos. O prolongamento do intervalo Q-T pode ocorrer com o uso associado de cetoconazol e domperidona ${ }^{(15)}$. Esses dados são motivos de preocupações, uma vez que, na publicação de Clara(11), a droga demonstrou ação mais consistente, diminuindo regurgitações e vômitos quando a dose foi duplicada

A metoclopramida é um agente antidopaminérgico com efeitos colinérgicos e seratoninérgicos. A droga promove relaxamento do piloro, acelera o tempo de esvaziamento gástrico, aumenta o tônus do esfíncter esofágico inferior. A dose diária é de 0,5 a $1,0 \mathrm{mg} / \mathrm{kg}$, fracionada em duas ou três tomadas ${ }^{(16)}$. A metoclopramida deve ser prescrita com muito cuidado pelo pediatra, pois a dose terapêutica é próxima daquela em que se verificam efeitos colaterais relativos ao sistema nervoso central, tais como; sonolência, irritabilidade, reações distônicas e não raramente sintomas extrapiramidais ${ }^{(16)}$. 
$\mathrm{Na}$ revisão de Magalhães et $a^{(1)}$, foram citados apenas dois estudos com a metoclopramida e os resultados não foram consistentes quanto à ação da droga em comparação ao placebo $(n=71 ; R R=0,63$; IC $95 \%=0,07$ $5,71 ; p=0,68)$. Vale lembrar que, em função da retirada da cisaprida do mercado, nos últimos anos houve um aumento na prescrição de metoclopramida em alguns centros.

Craig et al, em 2006 ${ }^{(17)}$, publicaram uma metanálise com a revisão de sete ensaios clínicos que confrontaram a metoclopramida e o placebo em crianças. Em três estudos, a droga demonstrou eficácia na redução de sintomas e diminuição dos índices de refluxo ácido, sendo que nos demais ensaios não foi comprovada a eficácia da droga. $O$ risco de ocorrência de efeitos colaterais foi de $26 \%$ no grupo que recebeu a medicação. As evidências que apoiam a sua eficácia em DRGE são menores, embora um subgrupo de pacientes apresente benefícios quanto à redução de regurgitações e vômitos.

Lifchitz, em 2009(18), em revisão a respeito do tratamento medicamentoso antirrefluxo, aponta que a relação risco/benefício não é favorável ao uso da metoclopramida em crianças com refluxo gastroesofágico.

Houve apenas um estudo da literatura, publicado por Euler em $1980^{(19)}$ e revisado por Magalhães et a/(1), que empregou o Betanecol para tratamento do refluxo gastroesofágico em crianças. A pesquisa envolveu 45 pacientes, sendo 30 menores de um ano, e os dados obtidos apontaram redução dos sintomas e melhora do número de refluxos ácidos. A droga é um agonista colinérgico que atua aumentando diretamente a contração do esfíncter esofágico inferior. Há potencialmente muitos riscos de efeitos colaterais que limitaram novos estudos acerca da sua eficácia e segurança.

Guimarães et al, em 2006(20), publicaram uma revisão a respeito do tratamento das síndromes esofágicas e extraesofágicas decorrentes da DRGE, listando 12 indicações para tratamento antirrefluxo. Em apenas três indicações, a literatura aponta algum benefício do uso de procinéticos: lactente que não ganha peso e apresenta vômitos

\section{Referências bibliográficas}

1. Magalhães PV, Bastos TR, Appolinário JC, Bacaltchuk J, Mota Neto Jl. Revisão sistemática e metanálise do uso de procinéticos no refluxo gastroesofágico e doença do refluxo gastroesofágico em pediatria. Rev Paul Pediatr 2009;27:236-42

2. Hillemeier AC. Gastroesophageal reflux. Diagnostic and therapeutic approaches. Pediatr Clin North Am 1996;43:197-212.

3. Vandenplas Y, Belli D, Benhamou PH, Cadranel S, Cezard JP, Cucchiara S et al. Current concepts and issues in the management of regurgitation of infants: (procinético associado a um antiácido anti-H2); crianças com vômitos esporádicos de forma isolada e crianças com RGE associado a pneumonias recorrentes (associar o procinético à droga antissecretora anti-H2 ou inibidor de bomba de próton). Em todas as demais situações (sintomas de esofagite, queimação retroesternal, esofagite erosiva e não erosiva, epigastralgia, manifestações otorrrinolaringológicas, asma, tosse crônica, crises de apneias e cianose), o tratamento medicamentoso consiste no emprego de medicação antiácida anti-H2 ou inibidores de bomba de prótons.

Lifchtiz $^{(18)}$ considera que o emprego de procinéticos deva ser analisado com reservas. As medicações têm a propriedade de acelerar o esvaziamento gástrico e promover o aumento do tônus do esfíncter esofágico inferior, contribuindo para diminuir o número de refluxos ácidos e não ácidos. Os ensaios clínicos, até o momento, não confirmam essas ações de forma consistente e, portanto, as drogas não devem ser prescritas rotineiramente para o tratamento de crianças com DRGE (evidência grau IB). Tais medicamentos são indicados quando houver falhas do tratamento antiácido instituído, de forma associada e não isolada, e sempre com monitorização cuidadosa quanto aos efeitos colaterais.

Assim, as principais considerações quanto ao uso de procinéticos em Pediatria:

- Poucos estudos têm sido publicados utilizando os procinéticos atualmente disponíveis.

- A ação dessas drogas não é consistente, o que limita o seu uso isolado no tratamento da doença do refluxo gastroesofágico.

- Há riscos elevados de ocorrência de efeitos colaterais, especialmente com o uso da metoclopramida e bromoprida.

- Há necessidade de futuros estudos que envolvam um maior número de pacientes para que se possa firmar um conceito bem fundamentado a respeito do uso desse grupo de medicações.

\section{Mauro Sergio Toporovski ${ }^{1}$}

a reappraisal. Management guidelines from a working party. Acta Paediatr 1996;85:531-4.

4. Dent J, El-Serag HB, Wallander MA, Johansson S. Epidemiology of gastrooesophageal reflux disease: a systematic review. Gut 2005;54:710-7.

5. Nelson SP, Chen EH, Syniar GM, Christoffel KK. Prevalence of symptoms of gastroesophageal reflux during infancy. A pediatric practice-based survey. Pediatric Practice Research Group. Arch Pediatr Adolesc Med 1997;151: 569-72. 
6. Deal L, Gold BD, Gremse DA, Winter HS, Peters SB, Fraga PD et al. Agespecific questionnaires distinguish GERD symptom frequency and severity in infants and young children: development and initial validation. J Pediatr Gastroenterol Nutr 2005;41:178-85.

7. Gold BD. Is gastroesophageal reflux disease really a life-long disease: do babies who regurgitate grow up to be adults with GERD complications? Am J Gastroenterol 2006;101:641-4.

8. Rudolph CD, Mazur LJ, Liptak GS, Baker RD, Boyle JT, Colletti RB et al. Guidelines for evaluation and treatment of gastroesophageal reflux in infants and children: recommendations of the North American Society for Pediatric Gastroenterology and Nutrition. J Pediatr Gastroenterol Nutr 2001;32 (Suppl 2):S1-31

9. Augood C, MacLennan S, Gilbert R, Logan S. Cisapride treatment for gastrooesophageal reflux in children (Cochrane Review). In: The Cochrane Library, Issue 1, 2006. Oxford: Update Software; 2006.

10. Pritchard DS, Baber N, Stephenson T. Should domperidone be used for the treatment of gastro-oesophageal reflux in children? Systematic review of randomized controlled trials in children aged 1 month to 11 years old. $\mathrm{Br} \mathrm{J}$ Clin Pharmacol 2005;59:725-9.

11. Clara R. Chronic regurgitation and vomiting treated with domperidone. A multicenter evaluation. Acta Paediatr Belg 1979;32:203-7.

12. De Loore I, Van Ravensteyn H, Ameryckx L. Domperidone drops in the symptomatic treatment of chronic paediatric vomiting and regurgitation. A comparison with metoclopramide. Postgrad Med J 1979;55:40-2.
13. Bines JE, Quinlan JE, Treves S, Kleinman RE, Winter HS. Efficacy of domperidone in infants and children with gastroesophageal reflux. J Pediatr Gastroenterol Nutr 1992;14:400-5.

14. Carroccio A, lacono G, Montalto G, Cavataio F, Soresi M, Notarbartolo A. Domperidone plus magnesium hydroxide and aluminum hydroxide: a valid therapy in children with gastroesophageal reflux: a double-blind randomized study versus placebo. Scand J Gastroenterol 1994;4:300-4.

15. Collins KK, Sondheimer JM. Domperidone-induced QT prolongation: add another drug to the list. J Pediatr 2008;153:596-8.

16. Chicella MF, Batres LA, Heesters MS, Dice JE. Prokinetic drug therapy in children: a review of current options. Ann Pharmacother 2005;39:706-11.

17. Craig WR, Hanlon-Dearman A, Sinclair C, Taback S, Moffatt M. Metoclopramide, thickened feedings, and positioning for gastro-oesophageal reflux in children under two years (Cochrane Review). In: The Cochrane Library, Issue 1, 2006. Oxford: Update Software; 2006.

18. Lifschitz $\mathrm{CH}$ [homepage on the internet]. Management of gastroesophageal reflux disease in children and adolescents [cited 2009 July 14]. Available from: http://www.uptodate.com/patients/content/topic.do?topicKey= .oKISI8DOwq_mG

19. Euler AR. Use of bethanechol for the treatment of gastroesophageal reflux. $J$ Pediatr 1980;96:321-4.

20. Guimarães EV, Marguet C, Camargos PA. Treatment of gastroesophageal reflux disease. J Pediatr (Rio J) 2006;82 (Suppl 5):S133-45.
Instituição: Faculdade de Ciências Médicas da Santa Casa de São Paulo, São Paulo, SP, Brasil

${ }^{1}$ Responsável pela Disciplina de Gastroenterologia Pediátrica da Faculdade de Ciências Médicas da Santa Casa de São Paulo; Presidente do Departamento de Gastroenterologia da SPSP; Membro do Departamento de Gastroenterologia da Sociedade Brasileira de Pediatria, São Paulo, SP, Brasil

Endereço para correspondência:

Avenida Pacaembu, 1.083 - Santa Cecília

CEP 01234-010 - São Paulo/SP

E-mail: toporovski@uol.com.br

Recebido em: 10/5/09 